LAWRENCE LIVERMORE N A TION AL LABORATORY

\title{
Hohlraum-Driven Ignition-Like Double-Shell Implosions on the Omega Laser Facility
}

P. A. Amendt, H. F. Robey, H.-S. Park, R. E. Tipton,

J. L. Milovich, M. Bono, R. Hibbard, R. Wallace,

V. Yu Glebov

December 15, 2003

Physical Review Letters 
This document was prepared as an account of work sponsored by an agency of the United States Government. Neither the United States Government nor the University of California nor any of their employees, makes any warranty, express or implied, or assumes any legal liability or responsibility for the accuracy, completeness, or usefulness of any information, apparatus, product, or process disclosed, or represents that its use would not infringe privately owned rights. Reference herein to any specific commercial product, process, or service by trade name, trademark, manufacturer, or otherwise, does not necessarily constitute or imply its endorsement, recommendation, or favoring by the United States Government or the University of California. The views and opinions of authors expressed herein do not necessarily state or reflect those of the United States Government or the University of California, and shall not be used for advertising or product endorsement purposes. 


\title{
Hohlraum-driven ignition-like double-shell implosions on the Omega laser facility
}

\author{
Peter A. Amendt, Harry F. Robey, H.-S. Park, R.E. Tipton, R.E. Turner, J.L. Milovich, M. Bono, R. \\ Hibbard, H. Louis, R. Wallace \\ Lawrence Livermore National Laboratory, Livermore, California 94550 \\ V. Yu. Glebov \\ Laboratory for Laser Energetics, U. of Rochester
}

\begin{abstract}
High-convergence ignition-like double-shell implosion experiments have been performed on the Omega laser facility [T.R. Boehly et al., Opt. Commun. 133, 495 (1997)] using cylindrical gold hohlraums with 40 drive beams. For the first time, repeatable, dominant primary $(2.45 \mathrm{MeV})$ neutron production from the mix-susceptible compressional phase of a double-shell implosion, using fall-line design optimization and exacting fabrication standards, is demonstrated. Effective control of fuel-pusher mix during final compression is essential for achieving noncryogenic ignition with double-shell targets on the National Ignition Facility [Paisner et al., Laser Focus World 30, 75 (1994)].
\end{abstract}

PACS numbers: 52.40.Nk, 52.50.Jm, 52.58.Ns

The goal of inertial confinement fusion is to implode a capsule filled with deuterium-tritium (DT) to a sufficient density and temperature for achieving thermonuclear ignition and energy gain [1]. In the single-shell indirect-drive option, a capsule is placed at the center of a hollow high- $\mathrm{Z}$ radiation enclosure (or hohlraum) which converts absorbed laser rays into x rays that ablate the outside of the capsule and drive an implosion. This current mainline ignition option requires cryogenic preparation of the capsule near the triple point of deuterium $\left(18.3^{\circ} \mathrm{K}\right)$ and careful shock sequencing to maintain the fuel on a low enough adiabat for achieving thermonuclear burn and high gain [2]. The required laser power history for preforming the DT-ice pusher to sufficient density $\left(\approx 10^{3} \mathrm{~g} / \mathrm{cc}\right)$ has a high contrast (50-to-1), delivering high power at late time when the hohlraum has filled with plasma and potentially leading to harmful laser backscatter from parametric instabilities.

A complementary approach to demonstrating ignition utilizes noncryogenic double-shell targets where the dense, high-Z inner shell provides inertial confinement and radiation trapping. With less need for careful shock-timing, the requirements on the laser pulse-shape become more relaxed, allowing the option of a reverse-ramp power history and the prospect of reduced plasma-mediated laser backscatter. An added property of double-shell ignition is that the mode of thermonuclear burn occurs via volume ignition [3] 
rather than with $(10 \mathrm{keV})$ hot-spot ignition and the associated self-sustaining fusion detonation wave [2]. Although the thermonuclear yield (of several MJ) is comparatively low with current double-shell ignition target designs [4,5] for the National Ignition Facility (NIF) [6], the lower threshold ignition temperature of $\approx 4 \mathrm{keV}$ makes ignition easier to achieve by relaxing the requirements on implosion symmetry. However, the main caveat with double-shell ignition is the required control of potentially ruinous mix of high- $\mathrm{Z}$ pusher material and DT fuel to tolerably small levels.

The concept of double-shell ignition has been tested over the past 10 years with laser experiments on the Nova [7] and Omega [8] laser facilities, though with mixed success. A traditional metric of implosion performance is the ratio of the measured primary neutron yield $\left[\mathrm{D}+\mathrm{D} \rightarrow \mathrm{n}(2.45 \mathrm{MeV})+\mathrm{He}^{3}(0.82\right.$ $\mathrm{MeV})]$ to the calculated "clean" yield (in the absence of atomic mix between the fuel and pusher) [9], i.e., YoC. All-glass inner-shell double-shell experiments have consistently given YoCs of a few percent at most, thereby challenging our understanding of double-shell behavior. Recently, double-shell experiments were fielded on the Omega laser with hybrid glass/plastic inner shells that gave YoCs closer to unity [10]. A key feature of these experiments is that preheat M-band x-ray radiation (2-5 keV) from the laser-irradiated gold hohlraum walls causes the inner shell to expand appreciably before shell collision, thereby resulting in reduced neutron production from the critically important compressional or stagnation phase of the implosion. This implosion scenario skews the neutron production history towards the earlier episode of shock convergence at capsule center (or "shock-flash"), well before deleterious atomic mixing of the cold pusher and hot fuel has occurred. Despite a high YoC, such an "exploding pusher" mode is not ignitionlike since only a modest fraction of the neutrons $(<0.5)$ are produced after deceleration onset at the critically important compressional stage. A key goal of ongoing double-shell ignition research is to demonstrate appreciable control of fuel-pusher mix so that the vast majority of neutrons are produced during final compression - a prerequisite for achieving ignition [11]. In this Letter we demonstrate for the first time repeatable ignition-like double-shell behavior with compressional neutron yields overwhelming the shockflash component by $20-35 \times$ or more. Moreover, we show that the double-shell performance is comparable to the highest performing hohlraum-driven single-shell implosions to date [12] - and at higher fuel convergence [13]. This advance in double-shell performance was made possible by exacting target fabrication, careful laser power control, and physical design criteria geared to reducing the effects of fuelpusher atomic mix. 
The Omega indirect-drive double-shell experimental configuration is shown in Fig. 1. Three cones with 5, 5, and 10 beams, respectively, enter each end of the gold hohlraum through a $75 \%$ laser-entrance hole (LEH) at three distinct angles to the axis of symmetry: $\theta=21.4^{\circ}, 42^{\circ}$ and $58.9^{\circ}$. The $0.351 \mu \mathrm{m}$ wavelength radiation is absorbed in the gold hohlraum walls and re-radiated as a quasi-Planckian spectrum of $\mathrm{x}$ rays with a hard component at $2-5 \mathrm{keV}[n=4 \rightarrow 3]$. The thermal $\mathrm{x}$ rays $(<1 \mathrm{keV})$ are absorbed in the outer shell which consists of polystyrene $(\mathrm{CH})$ and $2 \%$ [at.] bromine doping for preheat control of the all$\mathrm{CH}$ inner shell. The ablating (and converging) outer shell then transfers an appreciable fraction $(\approx 1 / 2)$ of its kinetic energy to the inner shell and compresses the encapsulated deuterium fuel to thermonuclear conditions $(>1 \mathrm{keV})$.

Two principal design considerations were implemented in this double-shell implosion campaign. First, the goal was to have the compressional stage of (clean) neutron production greatly dominate the earlier shock-flash neutron burst in order to mimic the behavior of a proposed igniting double-shell on the NIF [5]. X-ray preheat control with mid-Z doping of the outer shell is a proven technique [9] for arranging such implosion behavior. The second (and related) goal is the control of mix between the fuel and pusher (induced by Rayleigh-Taylor instability growth) to ensure appreciable compressional neutron production. A useful figure-of-merit for controlling mix is the "fall-line" delay [5]. Physically, the fall-line refers to the trajectory of free-falling interfacial material after deceleration onset. From causality considerations little, if any, pusher material is expected ahead of the fall-line trajectory, so that an arranged large delay in the fallline trajectory to the capsule center relative to the instant of peak neutron burn gives added margin to mix. Thus, this second goal is tantamount to maximizing the fall-line delay in order to optimize neutron production following deceleration onset.

The double-shell target dimensions were specified to meet these two design goals [See Fig. 1], subject to the energy and power limitations on the requested reverse-ramp laser pulse-shape [See Fig. 2]. Strict fabrication requirements [14] were applied to ensure repeatable target performance. Micro-machined carbonized-resorcinal-formaldehyde foam hemispherical inserts were used to support the inner shell and meet the shell concentricity specification $(<5 \mu \mathrm{m})$. This foam material was chosen because of its favorable machining properties and inherently small pore size $(<100 \mathrm{~nm})$. A relatively small pore size provides a higher margin to the seeding of destructive hydrodynamic instability growth following shell collision [15]. The outer shell consisted of two hemispherical shells with a machined epoxy-filled step joint to ensure 
complete ( $4 \pi$ steradian) x-ray shielding of the inner shell. The assembled double-shell target was then sandwiched between two $\approx 600-1000 \AA$ thick Formvar ${ }^{\circledR}$ tents for mounting at the hohlraum center.

The laser pulse-shape was chosen to provide a nominal hohlraum drive temperature of $\approx 185 \mathrm{eV}$ up to $2.5 \mathrm{~ns}$. This temperature was monitored with an array of calibrated x-ray diodes (Dante [16]) viewing through the LEH at $37^{\circ}$ from the symmetry axis. Figure 2 shows that the comparison between measured and postprocessed two-dimensional (2D) radiation-hydrodynamics simulations is well within the measurement uncertainties. Full-aperture laser backscatter measurements on the outer two cones $\left(42^{\circ}, 58.9^{\circ}\right)$ indicate negligible levels $(<200 \mathrm{~J})$, as expected for this pulse-shape. Measured peak x-ray and neutron emission times from the imploded core agree with calculations and provide an additional check on the hohlraum drive at target center. The highest energy channel of Dante provides a temporal record of $2-5 \mathrm{keV}$ radiation exiting the LEH. Figure 3 shows a comparison between the measured and postprocessed M-band fraction, indicating good agreement at late time but a significant difference up to $1.5 \mathrm{~ns}$. To correct for this disagreement, the nominal non-local thermodynamic equilibrium calculation with shell-averaged $\mathrm{Au}$ opacities (XSN [17]) was phenomenologically adjusted with time-dependent Au emissivity opacity multipliers above $2 \mathrm{keV}$. As a consistency check, these multipliers were then applied to modeling a dedicated diagnostic target for measuring the M-band strength at target center. This double-shell diagnostic target was specifically designed to implode a CH-tamped glass inner shell with non-thermal (>1 keV) $\mathrm{x}$ rays alone by delaying shell collision with an oversized $2 \%$ Ti-doped $\mathrm{CH}$ outer shell. The trajectory of the imploding glass shell was inferred from 60ps gated $\mathrm{Cr} \mathrm{He}-\alpha(5.6 \mathrm{keV})$ backlit images. Figure 4 shows the measured and calculated trajectory of the inner shell transmission minimum with and without enhanced Mband x-ray emission. This independent (and integrated) measure of the M-band fraction confirms the high level of early time preheat seen with Dante and lends support to our phenomenological preheat analysis.

Constraining the x-ray drive is crucial for lending credibility to a simulated neutron yield. Figures 2-4 collectively argue for a fairly consistent understanding of the level of thermal and M-band x-ray drive in the experiment. The focus on M-band fraction is derived from the expected high sensitivity of an Omega-scale double-shell implosion to x-ray preheat. With the limited energy available on Omega for the prescribed pulse-shape [<16kJ (See Fig. 2)], the inner shell must be thin enough to reach a sufficiently high implosion velocity - but not so thin that feed-through of hydrodynamic instability from the outside surface to the inner surface leads to shell breakup. For such thin inner shells, the optical depth of a $2 \mathrm{keV}$ 
photon is less than unity and leads to volumetric expansion and reduced hydrodynamic efficiency. Figures 5(a-b) summarize the neutron performance for this Omega double-shell campaign. Figure 5(a) shows the observed yield compared to the simulated shock-flash yield for all six double-shell implosions. Of these targets, the first five met all fabrication specifications without exception. The first used a $13 \mu \mathrm{m}$ thick inner shell and 0.1atm Ar doping in the fuel for core imaging [See Fig. 1], while the next four targets used a nominally thicker inner shell $(17 \mu \mathrm{m})$ to provide added margin to potential shell breakup from perturbation feed-through. For each of these targets, the neutron yield far exceeds the shock-flash yield as designed. The final target (\#6) in Fig. 5(a) had a large azimuthal machining defect in the outer shell, but was patched with a closely density-matched epoxy and re-machined. The measured yield of this capsule is just above the level due to shock-flash, suggesting that additional mixing induced by the defect strongly degraded the compressional phase of the implosion. This "control" result underscores the critical importance of stringent fabrication requirements for ignition-like double shells.

Figure 5(b) compares the performance of the five double-shells that met the exacting fabrication specifications compared with the Omega cylindrical hohlraum implosion database. The suite of "HEP5" targets [12] refers to $1 \%$ Ge-doped $\mathrm{CH}$ single-shell implosions driven by a medium contrast ratio (5-to-1) pulse-shape. The three surrogate single-shell capsules were fielded along with the double shells to assess hohlraum radiation symmetry and to provide a direct single-shell comparison in performance for the reverse-ramp pulse-shape. The $\mathrm{YoC}_{2 \mathrm{D}}$ metric refers to the inclusion of calculable $2 \mathrm{D}$ intrinsic hohlraum radiation asymmetry effects on the simulated yield as well as $a \approx 3 \%$ systematic left-right laser power imbalance. The dimensionless fall-line parameter $\Delta \tau$ is defined as the peak burn time minus the fall-line time normalized to the FWHM burn width. As expected, the double-shell targets show more sensitivity to M-band preheat compared with the single-shell surrogates [18]. Despite the important effect of enhanced Mband preheat on double-shell performance $(\approx 2 \times$ reduction in simulated DD neutron yield), the calculated (clean) compressional neutron yield fraction remains above 99\%. With M-band enhancement, both surrogate single shells and the double-shell targets follow a slow decline in neutron performance with increasing (and deteriorating) fall-line parameter. Furthermore, the performance of the double-shell implosions is comparable to the highest convergence $(\approx 20)$ HEP5 capsules (5atm DD fill) to date, attaining a $\mathrm{YoC}_{2 \mathrm{D}}$ up to $35 \%$ for a calculated clean convergence $\approx 30$. 
Gated hard (3-5 keV continuum) x-ray argon self-emission imaging of the imploded fuel core was also successfully demonstrated on a double-shell target to assess symmetry. Figure 6(a,b) compares a surrogate and double-shell core image near peak emission taken with $10 \mu \mathrm{m}$ spatial and 60ps temporal resolution. The distortion of the self-emission $50 \%$ contour for the surrogate target is less than for the double shell, but its convergence $(\approx 20)$ is also significantly less. Overall, the shape of the double-shell implosion shows some core-splitting, but the integrity of the imploded fuel core is intact.

In summary, repeatable ignition-like hohlraum-driven double-shell implosion behavior was demonstrated for the first time on the Omega laser facility. High fractional compressional neutron yields and implosion performance on par with high-convergence single-shell implosions were observed. The consistency in performance with the fall-line parameter over a wide variety of target types and fuel convergences provides important validation of this metric as a mix-mitigation design tool for realizing double-shell ignition on the NIF.

We acknowledge useful discussions with Jeff Colvin, Nino Landen, Dana Rowley and Larry Suter. We are indebted to the Omega team for operational support. This work was performed under the auspices of the U.S. Department of Energy by the University of California, Lawrence Livermore National Laboratory under Contract No. W-7405-Eng-48.

\section{Figure Captions}

Fig. 1: Three-dimensional rendering of hohlraum and Omega laser geometry. Hohlraum length (radius) is 2500 (800) $\mu \mathrm{m}$; inner beams (red) cross symmetry axis at $\pm 1850 \mu \mathrm{m}$, intermediate beams (green) cross axis at $1400 \mu \mathrm{m}$, and outer beams (blue) cross at $1200 \mu \mathrm{m}$. Also shown is preshot radiograph of a double-shell implosion target with 2\%-Br-doped $\mathrm{CH}$ outer shell (o.d.=550 $\mu \mathrm{m}$, i.d.=446 $\mu \mathrm{m}$ ) and $\mathrm{CH}$ inner shell (o.d. $=244 \mu \mathrm{m}$, i.d. $=218 \mu \mathrm{m}$ ) containing $50 \mathrm{~atm}$ of deuterium and $0.1 \mathrm{~atm}$ of argon dopant.

Fig. 2: Measured (open squares) and simulated (solid) Dante drive temperature versus time; delivered total laser power history (solid) and requested power history versus time (dashed). 
Fig. 3: Measured (open squares) and simulated (dashed) Dante M-band flux fraction (2-5 keV) versus time. Also shown is phenomenologically matched Dante M-band fraction using time-dependent Au emission opacity multipliers above $2 \mathrm{keV}$ (solid).

Figs. 4: Measured (solid squares) and simulated $\mathrm{Cr} 5.6 \mathrm{keV}$ backlighter transmission minimum trajectory with (solid) and without (dashed-dotted) enhanced Au M-band fraction (2-5 keV) versus time [cf. Fig. 3]. Shown also is schematic of dedicated M-band diagnostic target; inner shell is web supported between two hemispherical butt-jointed outer shells.

Figs. 5(a-b): (a) Measured primary neutron yield (solid) and 2D simulated shock-flash neutron yield versus fielded double-shell implosion target; (b) observed-over-predicted primary neutron yielsd versus dimensionless fall-line parameter. In (b) former (HEP5) $1 \% \mathrm{Ge}$-doped $\mathrm{CH}$ single-shell data are shown in dark grey for indicated DD gas-fills; triangles denote single-shell surrogate capsules with (solid) and without (open) enhanced M-band radiation - squares indicate double-shell targets. Astericked targets had 0.1 atm argon dopant in fuel to facilitate $\mathrm{x}$-ray $(3-5 \mathrm{keV})$ core imaging; shown also is schematic of nominal surrogate capsule.

Fig. 6(a-b): Peak self-emission x-ray core image from imploded Ar-doped DD fuel for a surrogate singleshell capsule (a) and double-shell target (b); solid white contour denotes 50\% peak x-ray emission.

\section{References}

[1] J.D. Lindl, Inertial Confinement Fusion (Springer-Verlag, New York, 1998).

[2] S.W. Haan et al., Phys. Plasmas 2, 2480 (1995).

[3] H. Hora et al., J. Plasma Phys. 60(4), 743 (1998).

[4] D.B. Harris and W.S. Varnum, Bull Am. Phys. Soc. 41, 1479 (1996).

[5] P. Amendt et al., Phys. Plasmas 9(5), 2221 (2002).

[6] J.A. Paisner et al., Laser Focus World 30, 75 (1994).

[7] E.M. Campbell, Laaser Part. Beams 9, 209 (1991).

[8] T.R. Boehly et al., Opt. Commun. 133, 495 (1997). 
[9] O.L. Landen et al., Phys. Plasmas 5, 768 (1998).

[10] W.S. Varnum et al., Phys. Rev. Lett. 84, 5153 (2000).

[11] Compressional yields greater than shock-flash yields by at least a factor-of-100 are associated with significant charged particle $\left(\mathrm{He}^{4}\right)$ energy deposition in the fuel and onset of ignition.

[12] P. Amendt, R.E. Turner, and O.L. Landen, Phys. Rev. Lett. 89, 165001 (2002).

[13] We follow convention [Ref. 1] and understand fuel convergence to mean the initial ablator radius divided by the imploded fuel radius.

[14] R.L. Hibbard et al., Fusion Science Technology, to appear (2004).

[15] W. J. Garbett, private communication (2003).

[16] H.N. Kornblum, R.L. Kauffman, and J.A. Smith, Rev. Sci. Instrum. 57, 2179 (1986).

[17] W.A. Lokke and W.H. Grasberger, "XSNQ-U, a non-LTE emission and absorption coefficient subroutine," UCRL-52276, 1977; copies may be obtained from the National Technical Information Service, Springfield, VA 22161.

[18] The HEP5 single-shell database shows no comparable M-band anomaly principally because the forward-ramped pulse-shape that was used is associated with far less preheat at (computationally problematic) early time, cf. Fig. 3. 
Fig. 1

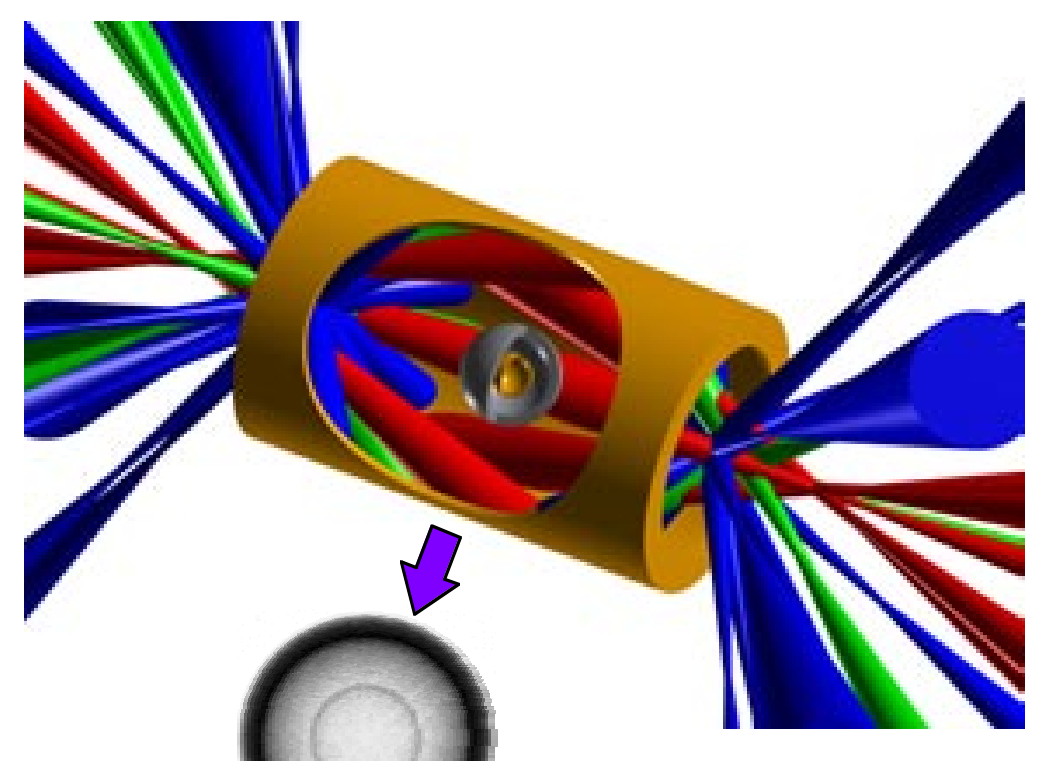


Fig. 2

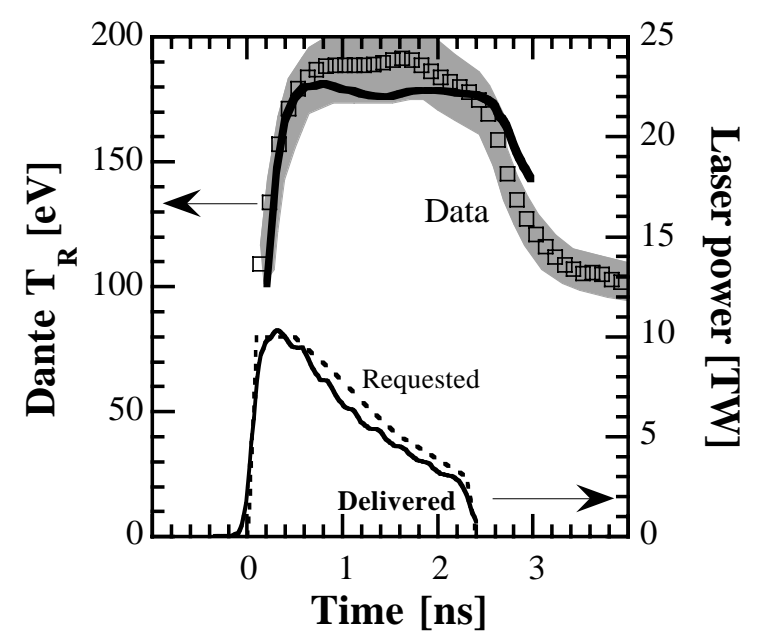


Fig. 3

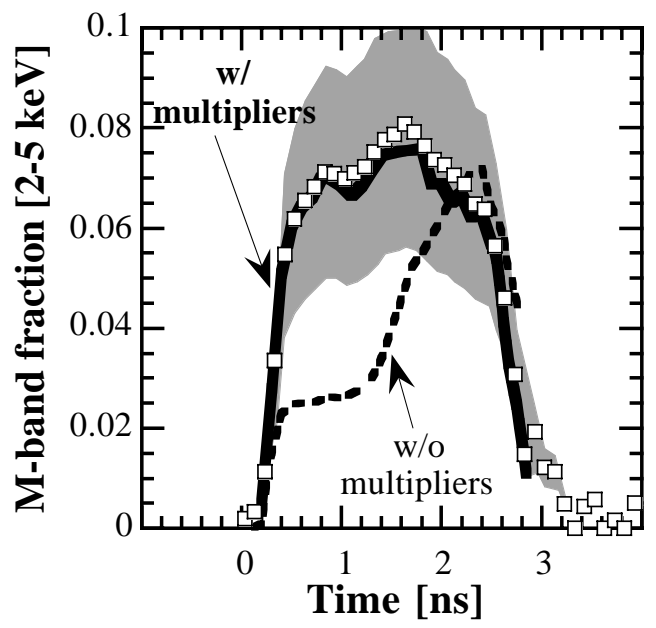


Fig. 4

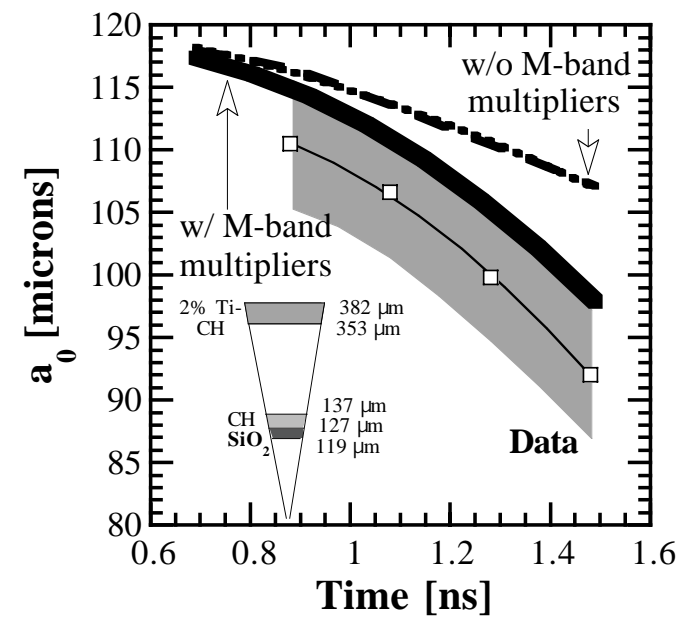


Fig. 5(a)

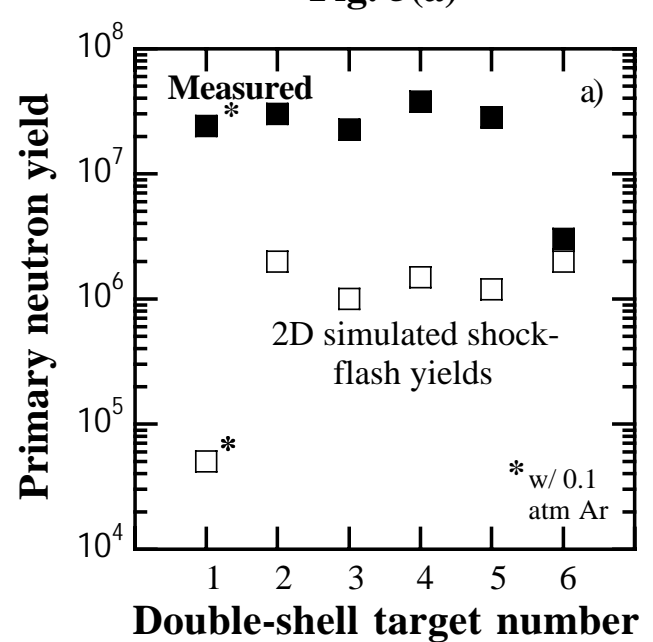


Fig. 5(b)

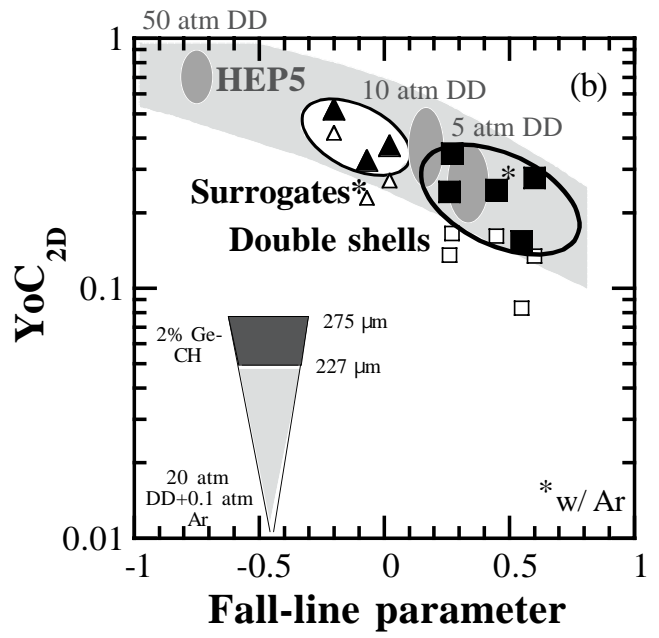


Fig. 6(a-b)

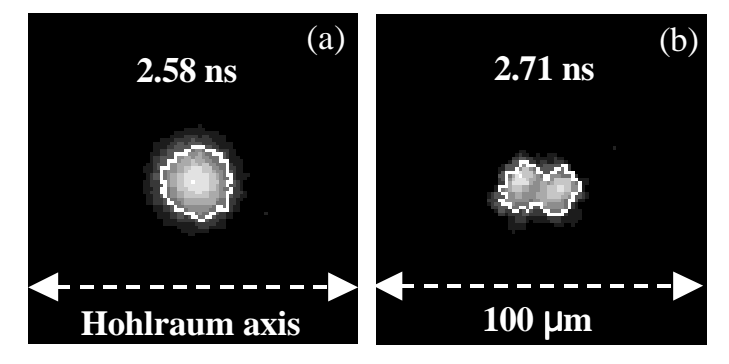

